\title{
To Compare the Morbidity and Mortality Pattern of late Preterm and Term Neonates at GRMC, Gwalior
}

\author{
${ }^{1}$ Neetu Sharma, ${ }^{2}$ Manish Rathore \\ ${ }^{1}$ Associate Professor, Department of Paediatrics, Gajara Raja Medical College, Gwalior, ${ }^{2}$ Senior Resident, Department of Paediatrics, Govt. Medical College, Ratlam.
}

\section{Abstract}

Background: This is a hospital based prospective observational study, carried out at Gajara Raja Medical College, Gwalior. All the inborn late preterm babies have been included in the study. Selection of late preterm neonates is done by determination of gestational age. Subjects and Methods: All the inborn late preterm babies have been included in the study. Selection of late preterm neonates is done by determination of gestational age. We compared the mortality and morbidity pattern of preterm neonates with the term counterpart, the data of term neonates retrieved from hospital record. Results: Maximum no. of cases in late preterm in Jaundice i.e. 9\%, followed by Septicaemia 7.8\% \&Respiratory Distress 7.6\%. In Term Jaundice i.e. 3.34\%, followed by Septicaemia 3.38\% \& Respiratory Distress 3.41\%. In our study Neonatal convulsions were more common among the late preterm neonates as compare to term group. Conclusion: In our study jaundice, septicemia, respiratory distress and hypoglycemia were found as major morbidities, among late preterm the rate was found to be $9 \%, 7.8 \%, 7.6 \%$ and $6 \%$ respectively while in term group the rate was $3.34 \%, 3.38 \%, 3.41 \%$ and $2.56 \%$ respectively. The mortality and morbidity among late preterm neonates were higher as compared to their term counterparts, implying the need for special attention to these babies because they may be overlooked among other preterm and sick babies.

Keywords: Morbidity, Mortality, Preterm \& Neonates.

Corresponding Author: Dr. Manish Rathore, Senior Resident, Department of Paediatrics, Govt. Medical College, Ratlam.

Received: July 2019

Accepted: July 2019

\section{Introduction}

The morbidity and mortality pattern in late preterm infants is higher than term infants (gestational age $\geq 37$ weeks). The main reason behind that is the relative physiologic and metabolic immaturity, though there is no significant difference in the weight or the size of the two groups. The late preterm infants are at twice to thrice increased risk of morbidities like hypoglycaemia, poor feeding, jaundice, infection and re-admission rates after initial hospital discharge. ${ }^{[1]}$ The infant mortality rate during first year of life for late-preterm infants is on an average four-fold higher than that for term infants. ${ }^{[2]}$

During the past few years proportion of late-preterm births has increased. The reason for the increase in late-preterm births during the last decade is not well understood. One hypothesis is that it may be attributable, in part, to increased use of reproductive technologies and, as a result, an increase in multi fetal pregnancies. Another hypothesis is that advances in obstetric practice have led to an increase in surveillance and medical interventions during pregnancy, as a result, at risk infants like those of intrauterine growth restrictions, fetal abnormalities and anomalies can be identified earlier and hence delivered earlier. ${ }^{[3]}$

The various morbidities of late preterm birth arise from immature organ systems that are not yet prepared to support life in the extra uterine environment. The risk of acute

neonatal illness decreases with gestational age, reflecting the fragility and immaturity of the brain, lungs, immune system, kidneys, skin, eyes, and gastrointestinal system. In general, more immature preterm infants require more life support. A Prospective study was conducted by Ezhilvannan NR, Vani HN;Niranjan HS et al, ${ }^{[4]}$ demonstrated short term adverse outcomes of late preterm neonates.

\section{Subjects and Methods}

This is a hospital based prospective observational study, carried out at Gajara Raja Medical College, Gwalior for a period of 12 months from Jan 2013 to Jan 2014.

\section{Inclusion criteria:}

Cases: All late preterm neonates (34-< 37 weeks of gestation) which were born at the Obstetrics department have been included.

Control: All term neonates which were born during the study period included as control group. 


\section{Sharma \& Rathore; Marlidity and Martality Pattern of late Preterm and Term Neanates}

\section{Exclusion criteria:}

Preterm babies ( $<34$ weeks of gestation)

Out born neonates which were admitted in SCNU and still births have been excluded.

\section{Method}

All the inborn late preterm babies have been included in the study. Selection of late preterm neonates is done by determination of gestational age. Gestational age is determined by using Naeglie's formula, antenatal ultrasound records and by applying Ballard scoring system on newborn. The babies were either shifted to NICU or to mother's side based on the baby's condition and was followed up till discharge or death. All the neonates were enrolled on a structured protocol, which included the data on antenatal care, maternal risk factors, mode and place of delivery, birth weight, gestational age, gender, diagnosis, relevant investigations, duration of stay and outcome. The data was recorded on Proforma and analyzed using descriptive statistics. Survival was defined as the discharge of a live infant from the hospital. Our study was to evaluate the short term outcomes of late preterm babies in comparison with term babies.

We compared the mortality and morbidity pattern of preterm neonates with the term counterpart, the data of term neonates retrieved from hospital record.

\section{Results \& Discussion}

Table 1: Comparison of various causes of morbidities between Late Preterm and Term Babies.

\begin{tabular}{|c|c|c|}
\hline \multirow[t]{2}{*}{ Neonatal Morbidities } & Late Preterm & Term \\
\hline & $($ Total $: \mathrm{N}=1301)$ & (Total: $\mathbf{N}=\mathbf{5 8 8 5}$ ) \\
\hline Jaundice & $117(9 \%)$ & $197(3.34 \%)$ \\
\hline Septicaemia & $101(7.8 \%)$ & $199(3.38 \%)$ \\
\hline Respiratory Distress & $99(7.6 \%)$ & $201(3.41 \%)$ \\
\hline Birth Asphyxia & $76(5.6 \%)$ & $117(2 \%)$ \\
\hline Hypoglycemia & $79(6 \%)$ & $15(2.56 \%)$ \\
\hline Feed Intolerance & $43(3.3 \%)$ & $98(1.6)$ \\
\hline Neonatal Convulsion & $42(3.22 \%)$ & $45(0.75 \%)$ \\
\hline Apnea & $39(3.03 \%)$ & $27(0.45 \%)$ \\
\hline NEC & $27(2.11 \%)$ & $13(0.22 \%)$ \\
\hline Congenital Malformation & $14(1.11 \%)$ & \begin{tabular}{|l|l|}
29 & $(0.5 \%)$ \\
\end{tabular} \\
\hline
\end{tabular}

Maximum no. of cases in late preterm in Jaundice i.e. 9\%, followed by Septicaemia7.8\% \&Respiratory Distress $7.6 \%$. In Term Jaundice i.e. 3.34\%, followed by Septicaemia3.38\% \&Respiratory Distress $3.41 \%$.

Table 2: Comparison of Occurrence of Neonatal Convulsion between Late Preterm and Term Babies.

\begin{tabular}{|l|l|l|l|}
\hline Neonatal & \multicolumn{2}{|l|}{ Gestational Maturity } & \multirow{2}{*}{ Total } \\
\cline { 1 - 3 } Convulsion & Late preterm (\%) & Term (\%) & \\
\hline Yes & $42(3.2)$ & $44(0.76)$ & 86 \\
\hline No & $1259(96.8)$ & $5841(99.24)$ & 7099 \\
\hline & 1301 & 5885 & 7186 \\
\hline
\end{tabular}

$\chi 2=109.5, \quad \mathrm{df}=1, \quad \mathrm{p}<0.000 \quad$ (difference is statistically significant)
$\mathrm{RR}=2.74,95 \% \mathrm{CI}=2.34,3.20$

In our study Neonatal convulsions were more common among the late preterm neonates as compare to term group. Late preterm's are at higher risk for neonatal morbidities and mortality contrary to the belief that they are nearly mature. The aim of the study was to evaluate short term outcome of late preterm's as compared with term gestation. This is a prospective observational study done over a period of 12 months. ${ }^{[5]}$

Out of the total live births, term births (37 weeks /more) were $75.99 \%$, late preterm births were $16.8 \%$ and preterm birth below 34 weeks were $7.2 \%$. It indicate $\mathrm{s}$ that late Preterm constitutes significant proportion of total birth. There is increase in proportion of late preterm neonates similar to study in USA whereas the proportion of late preterm babies has been increased from $6.2 \%$ in 1995 to 7.5 $\%$ in 2008, these changes are results of early obstetric interventions, ${ }^{[6]}$ for examples early termination of pregnancy in eclampsia, and other maternal and fetal morbidities. ${ }^{[7]}$

\section{Risk Factors, Morbidity And Mortality Data Of Late Preterm Neonates}

The various morbidities of late preterm birth arise from immature organ systems that are not yet prepared to support life in the extra uterine environment. The risk of acute neonatal illness decreases with gestational age, reflecting the fragility and immaturity of the brain, lungs, immune system, kidneys, skin, eyes, and gastrointestinal system. In general, more immature preterm infants require more life support. A Prospective study was conducted by Ezhilvannan NR, Vani HN;

Niranjan HS et $\mathrm{al}^{8}$ demonstrated short term adverse outcomes of late preterm neonates.

144 LPT comprised the study group. Male preponderance was present. Majority of the neonates had birth weight more than $2 \mathrm{~kg}$. PPROM and previous history of caesarian section formed the major maternal risk factor for preterm. Neonatal hyperbilirubinaemia was the major morbidity followed by respiratory distress, sepsis and feed intolerance. Majority of late preterm neonates required more than 7 days of hospital stay. Rate of rehospitalisation were also high among late preterm. Conclusion of the study was that late preterm had morbidity and mortality higher than expected; hence they need special attention while in hospital and a better follow up protocol after discharge. Late preterm infants comprise the majority of preterm newborns, caring for such a large population who are prone to have unfavorable outcomes can exert a profound impact on the society.

A prospective study was conducted on a total of 161 pregnant women who delivered LPT neonates at Department of Obstetrics and Gynaecology, KLE S Dr. Prabhakar Kore Hospital, Belgaum,by Divyakala 
Karegoudar1, Arati Prabhu et $\mathrm{al}^{9}$. During study maternal and perinatal outcome were assessed. Most of the women 56 (34.78\%) were aged 22 to 25 years and mean age was 24.54 \pm 4.18 years. Primipara was noted in $85(52.80 \%)$ of the women and history of previous preterm pregnancy was present in $3.11 \%$ of women. ${ }^{10}$ Labour was indicated in 60 $(37.27 \%)$ of the women while in $101(62.73 \%)$ it was spontaneous. In those with indicated labor, 14 (36.84\%) were induced and $36.84 \%$ underwent vaginal delivery while $24(63.16 \%)$ had emergency LSCS. With regard to spontaneous labour, 67 (66.34\%) had vaginal delivery and $34(33.66 \%)$ had emergency LSCS. Post partum eclampsia and eclampsia were noted in 2(1.24\%) each. ${ }^{11}$ The incidence of late preterm birth was $61.68 \%$. Most of the babies (41.61\%) had birth weight between 1.51 to $2.00 \mathrm{Kgs}$ and mean birth weight was $2.19 \pm 0.48 \mathrm{Kg}$. There were 84 $(52.17 \%)$ of babies who required NICU admission and low birth weight $51(60.71 \%)$ was the commonest cause. ${ }^{12}$ The mortality was observed in $5(5.95 \%)$ of the babies. Late preterm births being a large sub-group of preterm makes significant impact on perinatal outcome at each week of gestation 34, 35 and 36 weeks 6 days respectively. Hence managing late preterm births needs judicious decision making to reduce the mortality and morbidity. ${ }^{[13-15]}$.

\section{Conclusion}

In our study jaundice, septicemia, respiratory distress and hypoglycemia were found as major morbidities, among late preterm the rate was found to be $9 \%, 7.8 \%, 7.6 \%$ and $6 \%$ respectively while in term group the rate was $3.34 \%, 3.38 \%$, $3.41 \%$ and $2.56 \%$ respectively.

The mortality and morbidity among late preterm neonates were higher as compared to their term counterparts, implying the need for special attention to these babies because they may be overlooked among other preterm and sick babies.

\section{References}

1. Escobar GJ, McCormick MC, Zupancic JA, et al. Unstudied infants: Outcomes of moderately premature infants in the NICU. Paediatric Research. 2004;55:505A.

2. Pulver LS, Guest-Warnick G, Stoddard GJ, Byington CL, Young PC. Weight for gestational age affects the mortality of late preterm infants. Paediatrics. 2009;123(6).

3. Hankins GD, Longo M. The role of stillbirth prevention and late preterm (nearterm) births. SeminPerinatol. 2006;30:20-23

4. Ezhilvannan NR, Vani HN, Niranjan HS, Naveen Benakappa et al, Clinical profile and early hospital outcome of late preterms admitted in a tertiary care neonatal unit from South India. International Journal of Contemporary Pediatrics, Int J ContempPediatr. 2015; 2(3): 216-220.

5. Bhutani VK, Johnson L. Kernicterus in late preterm infants cared for as term healthy infants. SeminPerinatol 2006;30: 89-97.

6. Watchko JF. Hyperbilirubinemia and Bilirubin toxicity in the late preterm infant.,ClinPerinatol 2006;33:839-52.

7. Adams-Chapman I. Neurodevelopmental outcome of the late preterm infant. ClinPerinatol 2006;33:947-64.

8. Ezhilvannan NR, Vani HN, Niranjan HS, Naveen Benakappa et al, Clinical profile and early hospital outcome of late preterms admitted in a tertiary care neonatal unit from South India. International Journal of Contemporary Pediatrics, Int J Contemp Pediatr. 2015; 2(3): 216-220.

9. Divyakala Karegoudar1, Arati Prabhu2Perinatal Outcome and Associated maternal Co-morbid conditions in late Preterm Births - A Prospective study at Kles Dr. Prabhakar Kore Hospital, Belgaum, India, ISSN: 2319-7706 Volume 3 Number 6 (2014) pp. 865-875).

10. Watchko JF. Hyperbilirubinemia and bilirubin toxicity in the late preterm infant., Clin Perinatol 2006;33:839-52.

11. Adams-Chapman I. Neurodevelopmental outcome of the late preterm infant. Clin Perinatol 2006;33:947-64.

12. Moster D, Lie RT, Markestad T. Long-term medical and social consequences of preterm birth. N Engl J Med 2008;359:262-273.

13. Bhushan V, Paneth N, Kiely JL. Impact of improved survival of very low birth weight infants on recent secular trends in the prevalence of cerebral palsy, Pediatrics 1993;91:1094-1100

14. Ballard JL, Khoury JC, Wedig K, et al: New Ballard Score, expanded to include extremely premature infants. J Pediatrics 1991; 119:417-423.

15. 15.Bhutani VK, Johnson L. Kernicterus in late preterm infants cared for as term healthy infants. Semin Perinatol 2006;30: 89-97.

Copyright: (C) the author(s), 2019. It is an open-access article distributed under the terms of the Creative Commons Attribution License (CC BY 4.0), which permits authors to retain ownership of the copyright for their content, and allow anyone to download, reuse, reprint, modify, distribute and/or copy the content as long as the original authors and source are cited.

How to cite this article: Sharma N, Rathore M. To Compare the Morbidity and Mortality Pattern of late Preterm and Term Neonates at GRMC, Gwalior. Asian J. Clin. Pediatr. Neonatol. 2019;7(3):9-11.

DOI: dx.doi.org/10.21276/ajcpn.2019.7.3.3

Source of Support: Nil, Conflict of Interest: None declared. 